\title{
COMPUTER-CONTROLLED VARIABLE-STRUCTURE SYSTEMS
}

\author{
$\mathrm{XINGHUO} \mathrm{YU}^{1}$ and RENFREY B. POTTS ${ }^{2}$
}

(Received 30 October 1990; revised 21 January 1991)

\begin{abstract}
A theory is developed for the computer control of variable-structure systems, using periodic zero-order-hold sampling. A simple two-dimensional system is first analysed, and necessary and sufficient conditions for the occurrence of pseudosliding modes are discussed. The method is then applied to a discrete model of a cylindrical robot. The theoretical results are illustrated by computer simulations.
\end{abstract}

\section{Introduction}

The theory and design of computer-controlled systems has recently become an active area of research in modern control theory [1]. A common situation in computer control is that the digital-analogue converter is constructed so that it holds the analog signal constant until a new conversion is demanded. It is then natural to choose the sampling instants at the times when the control changes. This so-called zero-order-hold sampling of a system leads to mathematical models in the form of difference equations. As is usual, the sampling is taken to be periodic.

Variable structure control with sliding modes has been used in many different fields of applications, and in recent work [6] a mathematical model approach to the problem of discrete variable structure control with pseudosliding modes has been developed. It is the main purpose of the present paper to apply this approach to the computer control of variable-structure systems using zero-order-hold periodic sampling. A simple essentially-linear

\footnotetext{
${ }^{1}$ Department of Math. and Computing, University of Central Qld., Qld., 4702.

${ }^{2}$ Applied Mathematics Department, The University of Adelaide, South Australia, 5001.

(C) Copyright Australian Mathematical Society 1992, Serial-fee code 0334-2700/92
} 
two-dimensional system is first analysed to illustrate the method. For this system, the analysis is exact, in the sense that the difference equations of the model are not just approximations to differential equations, but directly give the values of the state variables and the outputs at the sampling instants, on the assumption that the control signal is constant during the sampling intervals. The general method is then applied to a more complicated system-a discrete mathematical model of a cylindrical robot which has been proposed by Neuman and Tourassis [4]. The system is an example of a practical highlynonlinear system and the difference equations of the model are approximations to the differential equations. The theoretical results for both systems are illustrated by computer simulations.

\section{Continuous two-dimensional variable-structure system}

Consider the variable-structure system given in state-space form as the ordinary differential equations

$$
\begin{aligned}
& \dot{x}_{1}=x_{2}, \\
& \dot{x}_{2}=-f x_{2}+u
\end{aligned}
$$

with control law given by

$$
u=-\psi x_{1}
$$

with $\psi$ defined by

$$
\psi= \begin{cases}a & \text { if } x_{1}\left(c x_{1}+x_{2}\right) \geq 0 \\ -a & \text { if } x_{1}\left(c x_{1}+x_{2}\right)<0\end{cases}
$$

Without loss of generality, the constants $a, c$ are taken to be positive. This system is a linear system apart from the variable structure giving

$$
s=s(\mathbf{x})=c x_{1}+x_{2}=0
$$

as a possible sliding line.

It is well-known [2] that the necessary and sufficient conditions for $s(\mathbf{x})=0$ to be a sliding line, characterised by $\dot{x}_{1}=-c x_{1}$, are

$$
\lim _{s \rightarrow+0} \dot{s}<0, \quad \lim _{s \rightarrow-0} \dot{s}>0 .
$$

Since

$$
\lim _{s \rightarrow \pm 0} \dot{s}=x_{1}\left(c f-c^{2} \mp a\right),
$$

there exists a stable sliding mode for $a>0$ and $c>0$ provided that 


$$
c^{2}-a<c f<c^{2}+a .
$$

A necessary and sufficient condition for the sliding line to be reachable [3] is that $|f|<2 \sqrt{a}$.

In matrix form, (1) and (2) can be written

$$
\dot{\mathbf{x}}=\mathbf{A x}+\mathbf{b} u
$$

where

$$
\mathbf{A}=\left[\begin{array}{cc}
0 & 1 \\
0 & -f
\end{array}\right]
$$

and

$$
\mathbf{b}=\left[\begin{array}{ll}
0 & 1
\end{array}\right]^{\top} \text {. }
$$

\section{Discrete two-dimensional system}

3.1 Computer control. Instead of the continuous system we now consider a discrete system corresponding to computer control using zero-order-hold periodic sampling. Let $\mathbf{x}(k)$ denote $\mathbf{x}$ evaluated at periodic time intervals $k h$, where $k$ is an integer and $h$ is the constant sampling period. It follows from the zero-order-hold sampling that [1]

$$
\mathbf{x}(k+1)=\exp (\mathbf{A} h) \mathbf{x}(k)+\left[\int_{0}^{h} \exp (\mathbf{A} \tau) \mathbf{b} d \tau\right] u(k), \quad k=0,1, \ldots,
$$

since $u$ is constant in the sampling interval.

We now take into account the nonlinear variable structure represented by (3) and (4). With the usual convention that the control signal is continuous from the right, the behaviour in the interval $(k h,(k+1) h]$ is governed by the sampled control $u(k)$ given by

$$
u(k)= \begin{cases}-a x_{1}(k) & \text { if } x_{1}(k)\left(c x_{1}(k)+x_{2}(k)\right) \geq 0, \\ a x_{1}(k) & \text { if } x_{1}(k)\left(c x_{1}(k)+x_{2}(k)\right)<0 .\end{cases}
$$

From (10),

$$
\exp (\mathbf{A} h)=\left[\begin{array}{cc}
1 & \left(1-e^{-f h}\right) / f \\
0 & e^{-f h}
\end{array}\right]
$$

and

$$
\int_{0}^{h} \exp (\mathbf{A} \tau) \mathbf{b} d \tau=\left[(h / f)+\left(e^{-f h}-1\right) / f^{2}\left(1-e^{-f h}\right) / f\right]^{\top} .
$$

Thus

$$
\mathbf{x}(k+1)=\Phi^{ \pm}(h) \mathbf{x}(k)
$$


where

$$
\begin{aligned}
\boldsymbol{\Phi}^{ \pm}(h) & =\left[\begin{array}{ll}
\phi_{11}^{+}(h) & \phi_{12}^{ \pm}(h) \\
\phi_{21}^{ \pm}(h) & \phi_{22}^{ \pm}(h)
\end{array}\right] \\
& =\left[\begin{array}{cc}
1 \mp a\left[(h / f)+\left(e^{-f h}-1\right) / f^{2}\right] & \left(1-e^{-f h}\right) / f \\
\mp a\left(1-e^{-f h}\right) / f & e^{-f h}
\end{array}\right] .
\end{aligned}
$$

In accord with the variable structure, the appropriate sign to be chosen in these expressions is decided as follows:

$$
\begin{array}{rll}
\text { use top sign } & \text { if } & x_{1}(k) s(k) \geq 0 \\
\text { use bottom sign } & \text { if } & x_{1}(k) s(k)<0
\end{array}
$$

where

$$
s(k)=c x_{1}(k)+x_{2}(k) .
$$

3.2 Conditions for pseudo-sliding mode. We first determine for the computer control of the system the discrete analogue of the necessary and sufficient conditions. A necessary condition for the occurrence of a stable pseudosliding mode is that the trajectories approach the switching line $s=0$ from both sides. This implies [6] that if $s(k)=0$ and $x_{1}(k)$ and $s(k+1)$ are of opposite signs, then the top sign in (18) must be used. On the other hand, if $s(k)=0$ and $x_{1}(k)$ and $s(k+1)$ are of the same sign, then the bottom sign in (18) must be used.

From (21) it follows that

$$
s(k)=\left[\begin{array}{ll}
c & 1
\end{array}\right] \mathbf{x}(k),
$$

and hence from (16) that

$$
s(k+1)=\left[\begin{array}{ll}
c & 1
\end{array}\right] \Phi^{ \pm}(h) \mathbf{x}(k) .
$$

For $s(k)=0$, this yields

$$
s(k+1)=\left[\begin{array}{ll}
c & 1
\end{array}\right] \Phi^{ \pm}(h)\left[\begin{array}{ll}
1 & -c
\end{array}\right]^{\top} x_{1}(k) .
$$

With the use of (18), it follows that

$$
s(k+1)=\sigma_{ \pm}(h) x_{1}(k)
$$

where

$$
\sigma_{ \pm}(h)=\mp a(c h / f)+(c f \mp a)(f-c)\left(1-e^{-f h}\right) / f^{2} .
$$

A necessary condition for the existence of a stable pseudo-sliding mode, namely that $x_{1}(k)$ and $s(k+1)$ are of opposite signs for the top sign in (18) and of the same sign for the bottom sign, is accordingly

$$
\sigma_{+}(h)<0, \quad \sigma_{-}(h)>0 .
$$


Since

$$
\sigma_{ \pm}(h)=\left(c f-c^{2} \mp a\right) h+O\left(h^{2}\right),
$$

the inequalities $(8)$ are obtained as a necessary condition. The condition can be made to mimic a discretisation of (7) by recalling that $s(k)=0$, so that (25) can be written

$$
[s(k+1)-s(k)] / h=\left[\left(c f-c^{2} \mp a\right)+O(h)\right] x_{1}(k) .
$$

The left-hand side of (29) approaches $\dot{s}$ as $h$ approaches zero, so that in the limit of the continuous system the condition (8) is correctly obtained.

A sufficient condition can be determined from the following argument. It is clear that the condition must involve a restriction on the magnitude of $h$, for this controls the sizes of the steps along a trajectory. What is required is that when a trajectory is being stepped out, a step across the switching line must be sufficiently small so that region which forces the return in the direction to the switching line is not over-stepped [6]. This region is bounded by the switching line $s(\mathbf{x})=c x_{1}+x_{2}=0$ and an asymptote line, which will be denoted by

$$
r(\mathbf{x})=d(h) x_{1}+x_{2}=0 .
$$

This line can be determined by considering

$$
\frac{x_{1}(k+1)}{x_{2}(k+1)}=\frac{x_{1}(k)}{x_{2}(k)}
$$

which gives, using (16),

$$
\frac{\phi_{11}^{ \pm}(h) x_{1}(k)+\phi_{12}^{ \pm}(h) x_{2}(k)}{\phi_{21}^{ \pm}(h) x_{1}(k)+\phi_{22}^{ \pm}(h) x_{2}(k)}=\frac{x_{1}(k)}{x_{2}(k)} .
$$

This leads to the homogeneous quadratic equation

$$
\phi_{12}^{ \pm}(h) x_{2}^{2}+\left[\phi_{11}^{ \pm}(h)-\phi_{22}^{ \pm}(h)\right] x_{1} x_{2}-\phi_{21}^{ \pm}(h) x_{1}^{2}=0,
$$

which simplifies to

$$
x_{2}^{2}+g_{ \pm}(h) x_{1} x_{2} \pm a x_{1}^{2}=0
$$

where

$$
g_{ \pm}(h)=f \mp a\left[h\left(1-e^{-f h}\right)^{-1}-f^{-1}\right] .
$$

For the top sign, the quadratic (33) represents two straight lines, two coincident lines or the single point, the origin, according as $g_{+}(h)^{2}$ is greater than, equal to, or less than $4 a$. 
For the bottom sign, the quadratic (33) represents two straight lines. The asymptote of present interest has the equation $r=d x_{1}+x_{2}=0$, where

$$
d=d(h)=0.5 g_{-}(h)+0.5 \sqrt{g_{-}(h)^{2}+4 a} .
$$

The limiting case occurs when a step is from the switching line to the asymptote, that is, from $s(k)=0$ to $r(k+1)=0$. As in the derivation of (23),

$$
r(k+1)=\left[\begin{array}{ll}
d(h) & 1
\end{array}\right]\left[\begin{array}{ll}
\phi_{11}^{+}(h) & \phi_{12}^{+}(h) \\
\phi_{21}^{+}(h) & \phi_{22}^{+}(h)
\end{array}\right]\left[\begin{array}{ll}
1 & -c
\end{array}\right]^{\top} x_{1}(k),
$$

which leads to

$$
r(k+1)=\left\{d(h)\left[\phi_{11}^{+}(h)-c \phi_{12}^{+}(h)\right]+\phi_{21}^{+}(h)-c \phi_{22}^{+}(h)\right\} x_{1}(k) .
$$

If

$$
d(h)\left[\phi_{11}^{+}(h)-c \phi_{12}^{+}(h)\right]+\phi_{21}^{+}(h)-c \phi_{22}^{+}(h)<0
$$

the return in the direction of the switching line does not occur. The condition $r(k+1)=0$ places an upper bound $H$ on the value of $h$ given by

$$
\left[f^{2}-a f H+(a-f c)\left(1-e^{-f H}\right)\right] d(H)=a f\left(1-e^{-f H}\right)+c f^{2} e^{-f H},
$$

where the top sign has been used. If this equation has more than one solution, the minimum solution is taken as the required upper bound.

\section{Cylindrical robot}

4.1 Continuous system. The cylindrical robot has three degrees of freedom, a radial movement signified by the position coordinate $r$, a rotation signified by the joint angle coordinate $\theta$, and a vertical movement corresponding to the position coordinate $z$. Because the $z$ degree of freedom is uncoupled from the $r$ and $\theta$ degrees of freedom it can be analysed separately and will hereafter be ignored. The $r$ and $\theta$ equations of motion of the robot are [4]:

$$
\begin{aligned}
& F_{r}(t)=m \dot{v}-(1 / 2) I^{\prime}(r) \omega^{2}, \\
& F_{\theta}(t)=(d / d t)[I(r) \omega]
\end{aligned}
$$


In these equations:

$$
\begin{aligned}
F_{r} & =\text { radial component external force, } \\
F_{\theta} & =\text { external torque } \\
m_{R} & =\text { mass of radial link, } \\
m_{L} & =\text { mass of payload at link tip, } \\
m & =m_{R}+m_{L}, \\
l & =\text { length of radial link, } \\
I(r) & =\text { moment of inertia column, arm, } \\
& =7 I_{c}+m_{R} l^{2} / 3+m r^{2}-m_{R} l r, \\
v & =\dot{r} \\
\omega & =\dot{\theta} .
\end{aligned}
$$

The equations of motion are two second-order coupled differential equations and, although seemingly simple, afford a good illustration of the complexity of robotic problems.

4.2 Discrete system. Following Neuman and Tourassis [4], we consider a discretisation of the system by letting $r(k)$, for example, denote $r$ evaluated at time $k h$, where $h$ is a discrete constant time interval. As usual we define the forward difference operator by

$$
\Delta r(k)=r(k+1)-r(k)
$$

The two second-order differential equations of motion (41), (42) are replaced by the four first-order difference equations [4]

$$
\begin{aligned}
F_{r}(k)= & m \Delta v(k) / h-(1 / 2)\{\Delta I[r(k)] / \Delta r(k)\} \\
& \times\{\omega(k) \omega(k+1)\}, \\
F_{\theta}(k)= & \Delta[\{I[r(k)]\}\{\omega(k)\}] / h, \\
\Delta r(k) / h= & (1 / 2)[v(k+1)+v(k)], \\
\Delta \theta(k) / h= & (1 / 2)[\omega(k+1)+\omega(k)] .
\end{aligned}
$$

The last two equations are 'smoothing formulae' and, assuming the initial values $v(0)$ and $\omega(0)$ are known, enable subsequent values for $k=1,2,3, \ldots$ to be calculated from

$$
\begin{gathered}
v(k)=(-1)^{k} v(0)-2(-1)^{k} \sum_{i=0}^{k-1}(-1)^{i} \Delta r(i) / h, \\
\omega(k)=(-1)^{k} \omega(0)-2(-1)^{k} \sum_{i=0}^{k-1}(-1)^{i} \Delta \theta(i) / h .
\end{gathered}
$$


The important implication of these results is that $v(k)$ is a simple linear function of $r(0), r(1), \ldots, r(k)$ and similarly for $\omega(k)$. This enables the whole analysis to be based on position coordinates $r$ and $\theta$ without any use of velocities.

The fundamental property of this Neuman-Tourassis discretisation is that for zero-order-hold sampling, conservation of energy is maintained and for any cyclic or ignorable variable the corresponding impulse equation is satisfied.

For our purposes, we write (53)-(56) in matrix form as

$$
\begin{aligned}
\mathbf{R}_{k+1} \mathbf{r}(k+1) & =\mathbf{R}_{k} \mathbf{r}(k)+\mathbf{d}_{k}+\mathbf{b} h F_{r}(k), \\
\boldsymbol{\Theta}_{k+1} \boldsymbol{\theta}(k+1) & =\boldsymbol{\theta}_{k} \boldsymbol{\theta}(k)+\mathbf{b} h F_{\theta}(k),
\end{aligned}
$$

where

$$
\begin{aligned}
\mathbf{R}_{k+1} & =\left[\begin{array}{cc}
1 & -h / 2 \\
-m h \omega(k) \omega(k+1) / 2 & m
\end{array}\right], \\
\mathbf{R}_{k} & =\left[\begin{array}{cc}
1 & h / 2 \\
m h \omega(k) \omega(k+1) / 2 & m
\end{array}\right], \\
\mathbf{d}_{k} & =\left[\begin{array}{ll}
0 & -m_{R} l h \omega(k) \omega(k+1) / 2
\end{array}\right]^{\top}, \\
\mathbf{b} & =\left[\begin{array}{ll}
0 & 1
\end{array}\right]^{\top}, \\
\mathbf{r}(k) & =\left[\begin{array}{ll}
r(k) & v(k)
\end{array}\right]^{\top}, \\
\boldsymbol{\theta}_{k+1} & =\left[\begin{array}{cc}
1 & -h / 2 \\
0 & I[r(k+1)
\end{array}\right], \\
\boldsymbol{\theta}_{k} & =\left[\begin{array}{cc}
1 & h / 2 \\
0 & I[r(k)
\end{array}\right], \\
\boldsymbol{\theta}(k) & =\left[\begin{array}{ll}
\theta(k) & \omega(k)]^{\top} .
\end{array}\right.
\end{aligned}
$$

4.3 Switching surfaces. In the continuous case, the switching surfaces for VSS control usually take the form

$$
s=s(t)=\dot{x}+c x=0,
$$

where $x$ is a position signal, $\dot{x}$ a velocity signal, and $c$ is a chosen positive time constant.

For the discrete model of the cylindrical robot, there will be two switching surfaces for the VSS control, and these we choose as

where

$$
\begin{gathered}
s_{r}(k)=\mathbf{c}_{r}^{\top} \mathbf{r}(k)=v(k)+c_{r} r(k), \\
s_{\theta}(k)=\mathbf{c}_{\theta}^{\top} \boldsymbol{\theta}(k)=\omega(k)+c_{\theta} \theta(k),
\end{gathered}
$$

$$
\begin{aligned}
\mathbf{c}_{r} & =\left[\begin{array}{ll}
c_{r} & 1
\end{array}\right]^{\top} \\
\mathbf{c}_{\theta} & =\left[\begin{array}{ll}
c_{\theta} & 1
\end{array}\right]^{\top} .
\end{aligned}
$$

The time constants $c_{r}$ and $c_{\theta}$ are positive. 
4.4 Hierarchical zero-order-sampling control. Young [9] has proposed for multi-dimensional systems hierarchical control schemes consisting of sequences of two-dimensional controls, and we shall use this concept to develop a hierarchical zero-order-sampling control for the Neuman-Tourassis discrete model of the cylindrical robot. First, a variable structure control is used to force the system to chatter close to the switching surface $s_{\theta}(k)=0$ and then a subsequent control to force the chattering close to $s_{r}(k)=0$.

The details of this hierarchical control will now be described. Using (59) and $(60)$ we obtain

$$
\begin{aligned}
\Delta s_{r}(k) & =s_{r}(k+1)-s_{r}(k)=\mathbf{c}_{r}^{\top}[\mathbf{r}(k+1)-\mathbf{r}(k)] \\
& =\mathbf{c}_{r}^{\top}\left[\left(\mathbf{R}_{k+1}^{-1} \mathbf{R}_{k}-\mathbf{I}\right) \mathbf{r}(k)+\mathbf{R}_{k+1}^{-1} \mathbf{d}_{k}\right]+\mathbf{c}_{r}^{\top} \mathbf{R}_{k+1}^{-1} \mathbf{b} h F_{r}(k),
\end{aligned}
$$

and similarly

$$
\Delta s_{\theta}(k)=\mathbf{c}_{\theta}^{\top}\left[\boldsymbol{\Theta}_{k+1}^{-1} \boldsymbol{\Theta}_{k}-\mathbf{I}\right] \boldsymbol{\theta}(k)+\mathbf{c}_{\theta}^{\top} \boldsymbol{\Theta}_{k+1}^{-1} \mathbf{b} h F_{\theta}(k) .
$$

The control forces are now defined as sums of equivalent and variable control terms as follows:

$$
\begin{aligned}
& F_{r}(k)=F_{r}^{E}(k)+F_{r}^{V}(k), \\
& F_{\theta}(k)=F_{\theta}^{E}(k)+F_{\theta}^{V}(k) .
\end{aligned}
$$

Here $F_{r}^{E}(k), F_{\theta}^{E}(k)$ are the equivalent control forces chosen as

$$
\begin{aligned}
& F_{r}^{E}(k)=-\left(h \mathbf{c}_{r}^{\top} \mathbf{R}_{k+1}^{-1} \mathbf{b}\right)^{-1} \mathbf{c}_{r}^{\top}\left[\left(\mathbf{R}_{k+1}^{-1} \mathbf{R}_{k}-I\right) \mathbf{r}(k)+\mathbf{R}_{k+1}^{-1} \mathbf{d}_{k}\right], \\
& F_{\theta}^{E}(k)=-\left(h \mathbf{c}_{\theta}^{\top} \boldsymbol{\theta}_{k+1}^{-1} \mathbf{b}\right)^{-1} \mathbf{c}_{\theta}^{\top}\left[\boldsymbol{\theta}_{k+1}^{-1} \mathbf{\theta}_{k}-I\right] \boldsymbol{\theta}(k),
\end{aligned}
$$

in order to obtain

$$
\begin{aligned}
\Delta s_{r}(k) & =\mathbf{c}_{r}^{\top} \mathbf{R}_{k+1}^{-1} \mathbf{b} h F_{r}^{V}(k) \\
& =\frac{h\left(1+h c_{r} / 2\right) F_{r}^{V}(k)}{m\left[1-\omega(k) \omega(k+1) h^{2} / 4\right]}, \\
\Delta s_{\theta}(k) & =\mathbf{c}_{\theta}^{\top} \boldsymbol{\theta}_{k+1}^{-1} \mathbf{b} h F_{\theta}^{V}(k) \\
& =\frac{h\left(1+h c_{\theta} / 2\right) F_{\theta}^{V}(k)}{I[r(k+1)]} .
\end{aligned}
$$

The variable structure control terms $F_{r}^{V}(k), F_{\theta}^{V}(k)$ are chosen, in generalisation of (13), as

$$
\begin{aligned}
& F_{r}^{V}(k)= \begin{cases}-a_{r} r(k) & \text { if } r(k) s_{r}(k) \geq 0, \\
a_{r} r(k) & \text { if } r(k) s_{r}(k)<0,\end{cases} \\
& F_{\theta}^{V}(k)= \begin{cases}-a_{\theta} \theta(k) & \text { if } \theta(k) s_{\theta}(k) \geq 0, \\
a_{\theta} \theta(k) & \text { if } \theta(k) s_{\theta}(k)<0,\end{cases}
\end{aligned}
$$

where $a_{r}$ and $a_{\theta}$ are taken as suitable positive constants. 
The hierarchical control is established by first taking $F_{r}(k)=F_{r}{ }^{E}(k)$ and then choosing $F_{\theta}(k)$ so that the system chatters close to the switching surface $s_{\theta}(k)=0$ until the condition

$$
\left|s_{\theta}(k)\right|<\varepsilon_{\theta}
$$

is satisfied, where $\varepsilon_{\theta}$ is a suitably chosen positive constant. The control is then changed to $F_{r}(k)=F_{r}^{E}(k)+F_{r}^{V}(k)$ until pseudo-sliding occurs on the intersection of $s_{\theta}(k)=0$ and $s_{r}(k)=0$.

There is, however, a difficulty in calculating the equivalent control terms $F_{r}^{E}(k)$ and $F_{\theta}^{E}(k)$ because, according to $(79)$ and (80), they require a knowledge of the not yet determined values of $r(k+1)$ and $\omega(k+1)$. The difficulty is overcome by using an iterative method [5], [8] in which, in accord with the hierarchical ordering, first $r(k+1)$ in $I[r(k+1)]$ is replaced by $r(k)+\Delta r(k-1)$. This enables an estimate of $\theta(k+1)$ and hence of $\omega(k+1)$ to be made and this in turn enables $\mathbf{r}(k+1)$ to be estimated. The estimated value of $r(k+1)$ can then be used in $I[r(k+1)]$ and the iteration repeated until a satisfactory convergence is reached. In practice, two or three iterations suffice.

4.5 Conditions for pseudo-sliding mode. In general, a necessary (but not sufficient) condition for the existence of a pseudo-sliding mode for a switching surface $s(k)=0$ of a discrete system is that there exists an $\varepsilon>0$ such that, for all $k$ for which $|s(k)|<\varepsilon$,

$$
s(k) \Delta s(k)<0 .
$$

In other words, if the switching surface corresponds to a pseudo-sliding mode, then there is a region close to the surface such that at each point of the trajectory, the next step is towards the surface.

For the discrete cylindrical robot model, the choices (85) and (86) of the variable structure control terms ensure that

$$
\begin{aligned}
& s_{r}(k) \Delta s_{r}(k)=-a_{r}\left|r(k) s_{r}(k)\right| \frac{h\left(1+h c_{r} / 2\right)}{m\left[1-\omega(k) \omega(k+1) h^{2} / 4\right]}, \\
& s_{\theta}(k) \Delta s_{\theta}(k)=-a_{\theta}\left|\theta(k) s_{\theta}(k)\right| \frac{h\left(1+h c_{\theta} / 2\right)}{I[r(k+1)]},
\end{aligned}
$$

where use has been made of (82) and (84). With the proviso that

$$
\omega(k) \omega(k+1) h^{2}<4,
$$

it follows that $s_{r}(k) \Delta s_{r}(k)<0$ and $s_{\theta}(k) \Delta s_{\theta}(k)<0$. 
A sufficient condition involving a restriction on the magnitude of $h$ can be determined from an argument similar to that in Section 3.2. The appropriate asymptote lines are obtained from

$$
\begin{aligned}
& \frac{r(k+1)}{v(k+1)}=\frac{r(k)}{v(k)}, \\
& \frac{\theta(k+1)}{\omega(k+1)}=\frac{\theta(k)}{\omega(k)} .
\end{aligned}
$$

Substituting (59) and (60) into (92) and (93), we get

$$
\begin{aligned}
\mathbf{r}(k+1) & =\mathbf{U}^{ \pm} \mathbf{r}(k), \\
\boldsymbol{\theta}(k+1) & =\mathbf{V}^{ \pm} \boldsymbol{\theta}(k),
\end{aligned}
$$

where

$$
\begin{aligned}
& \mathbf{U}^{ \pm}=\mathbf{R}_{k+1}^{-1}\left[\mathbf{R}_{k}-\left(\mathbf{c}_{r}^{\top} \mathbf{R}_{k+1}^{-1} \mathbf{b}\right)^{-1} \mathbf{b} \mathbf{c}_{r}^{\top}\left(\mathbf{R}_{k+1}^{-1} \mathbf{R}_{k}-I\right) \mp a_{r} h \mathbf{b e} \mathbf{e}^{\top}\right] \\
& \mathbf{V}^{ \pm}=\mathbf{\Theta}_{k+1}^{-1}\left[\boldsymbol{\Theta}_{k}-\left(\mathbf{c}_{\theta}^{\top} \boldsymbol{\Theta}_{k+1}^{-1} \mathbf{b}\right)^{-1} \mathbf{b} \mathbf{c}_{\theta}^{\top}\left(\mathbf{\Theta}_{k+1}^{-1} \boldsymbol{\Theta}_{k}-I\right) \mp a_{\theta} h \mathbf{b e} \mathbf{e}^{\top}\right]
\end{aligned}
$$

with $\mathbf{e}=\left[\begin{array}{ll}1 & 0\end{array}\right]^{\top}$. The elements of these matrices are given by

$$
\begin{aligned}
& u_{11}^{ \pm}=1 \mp \delta h^{2} a_{r} / 2, \\
& u_{12}^{ \pm}=\delta h m(1+\mu) / 2, \\
& u_{21}^{ \pm}=\mp \delta a_{r} h, \\
& u_{22}^{ \pm}=m \delta\left[\mu+\omega(k) \omega(k+1) h^{2} / 4\right], \\
& v_{11}^{ \pm}=1 \mp\left(h^{2} / 2\right) a_{\theta} / I[r(k+1)], \\
& v_{12}^{ \pm}=h(1-\nu) / 2, \\
& v_{21}^{ \pm}=\mp a_{\theta} h / I[r(k+1)], \\
& v_{22}^{ \pm}=-\nu
\end{aligned}
$$

with

$$
\begin{aligned}
& \mu=1-\left(1+c_{r} h / 2\right)^{-1}\left(c_{r} h+\omega(k) \omega(k+1) h^{2} / 2\right), \\
& \delta=m^{-1}\left[1-\omega(k) \omega(k+1) h^{2} / 4\right]^{-1}, \\
& \nu=\left(c_{\theta} h / 2-1\right)\left(c_{\theta} h / 2+1\right)^{-1} .
\end{aligned}
$$

The homogeneous quadratic forms of interest, and analogous to (33), are given using the bottom sign by

$$
\begin{aligned}
& u_{12}^{-} v^{2}(k)+\left(u_{11}^{-}-u_{22}^{-}\right) v(k) r(k)-u_{21}^{-} r^{2}(k)=0, \\
& v_{12}^{-} \omega^{2}(k)+\left(v_{11}^{-}-v_{22}^{-}\right) \omega(k) \theta(k)-v_{21}^{-} \theta^{2}(k)=0 .
\end{aligned}
$$


The required asymptotes have the equations

$$
\begin{gathered}
d_{r} r(k)+v(k)=0, \\
d_{\theta} \theta(k)+\omega(k)=0,
\end{gathered}
$$

where

$$
\begin{aligned}
& d_{r}=d_{r}(h)=0.5\left(u_{12}^{-}\right)^{-1}\left[u_{11}^{-}-u_{22}^{-}+\sqrt{\left(u_{22}^{-}-u_{11}^{-}\right)^{2}+4 u_{12}^{-} u_{21}^{-}}\right] \\
& d_{\theta}=d_{\theta}(h)=0.5\left(v_{12}^{-}\right)^{-1}\left[v_{11}^{-}-v_{22}^{-}+\sqrt{\left(v_{22}^{-}-v_{11}^{-}\right)^{2}+4 v_{12}^{-} v_{21}^{-}}\right] .
\end{aligned}
$$

A sufficient condition for the existence of a pseudo-sliding mode is accordingly that $h<H$ where

$$
\begin{aligned}
& H=\min \left\{H_{r}, H_{\theta}\right\}, \\
& H_{r}=\min _{\omega}\left\{h>0: u_{21}^{+}-c_{r} u_{22}^{+}+d_{r}\left(u_{11}^{+}-c_{r} u_{12}^{+}\right) \geq 0\right\}, \\
& H_{\theta}=\min _{I[r]}\left\{h>0: v_{21}^{+}-c_{\theta} v_{22}^{+}+d_{\theta}\left(v_{11}^{+}-c_{\theta} v_{12}^{+}\right) \geq 0\right\} .
\end{aligned}
$$

In evaluating these minima, use is made of the constraints on the magnitudes of $|r|,|v|,|\theta|,|\omega|$.

\section{Simulation results}

5.1 Two-dimensional system. For the simulation studies for the two-dimensional variable-structure system analysed in Section 2, the following values of the constants were chosen:

$$
c=1, \quad a=9, \quad f=-3.49, \quad x(0)=\left[\begin{array}{ll}
0.01 & 3
\end{array}\right]^{\top} .
$$

The corresponding upper bound of the stepsize is obtained from (40) as $H=0.0488$.

Figure 1 gives the simulation results showing the discrete points on the trajectories for two values of the stepsize. In both cases, the motion is stable, 


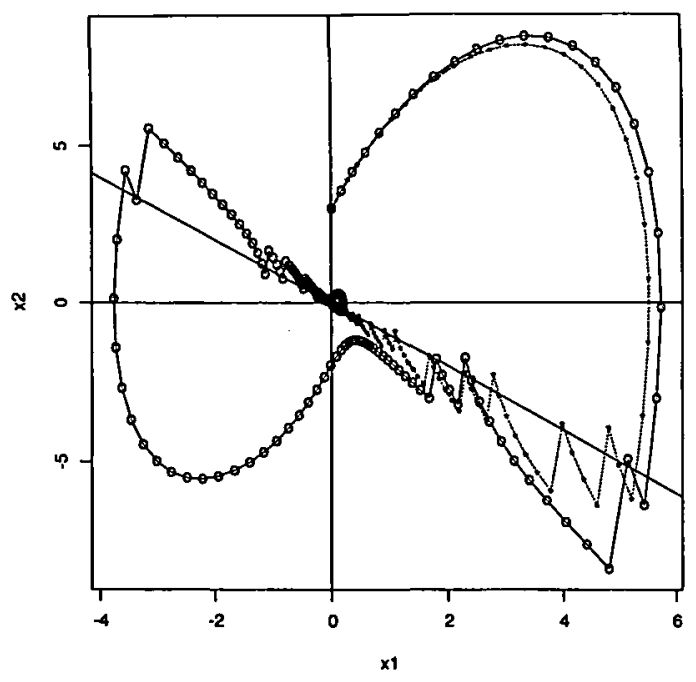

FIGURE 1. Discrete trajectories for the two-dimensional system. The discrete points represented by crosses and connected by the dashed lines signify the trajectory for a simpling interval $h=0.04$ which is less than the calculated upper bound $H=0.0488$ for the existence of a pseudo-sliding mode. For $h=0.05$, the trajectory is represented by the small circles connected by the solid lines.

the trajectories converging to the origin. In one case pseudo-sliding occurs, but not in the other.

For $h=0.04<H$, the discrete points being represented by small crosses joined by a dashed line, the existence of the pseudo-sliding mode is verified, with chattering occurring down the switching line $x_{1}+x_{2}=0$. It is evident that a condition that the trajectory moves towards the switching line from all points cannot be a necessary condition for the existence of a pseudo-sliding mode because the trajectory is seen to first move away before returning and remaining near the switching line. The fine structure of the trajectory is also of interest. Once the switching line is crossed, the trajectory returns towards it. But the extremities of a step crossing the switching line do not on each side of the line approach the switching line monotonically.

For $h=0.05>H$, pseudo-sliding does not occur, the trajectory alternately chattering along the switching line, and then swinging away from it, before returning.

Figure 2 (see page 14) illustrates the simulation results with the same data as for Figure 1 but with a smaller value $h=0.02$. Again the existence of the pseudo-sliding mode is verified. 


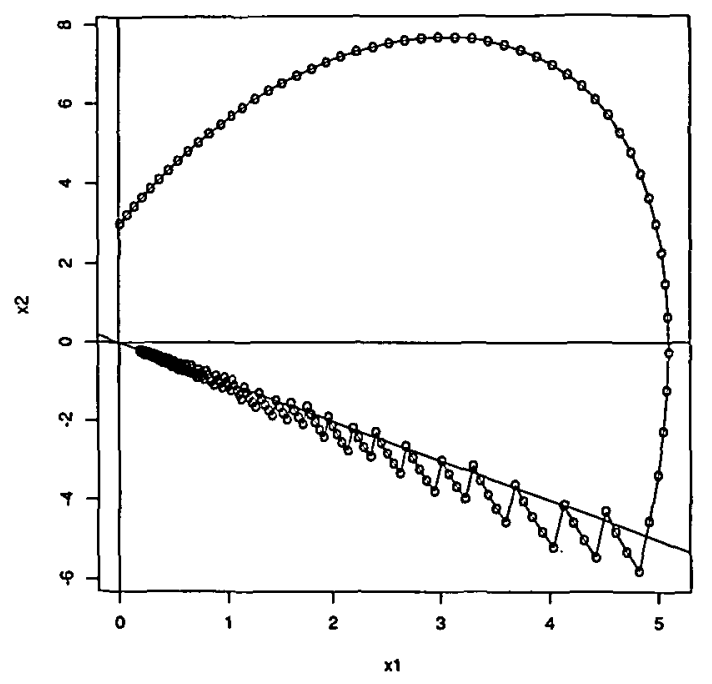

Figure 2. Discrete trajectory for the two-dimensional system. The same data as for Figure 1 but with $h=0.02$

5.2 Cylindrical robot. For the simulation studies for the cylindrical robot variable structure system analysed in Section 3, the following values of the constants were chosen:

$$
\begin{array}{cl}
m=7, & I(r)=10-2 r+7 r^{2}, \quad c_{r}=c_{\theta}=1, \\
& a_{r}=a_{\theta}=20, \quad \varepsilon_{\theta}=0.01 .
\end{array}
$$

In addition, the variables were limited by the constraints

$$
0<r<1, \quad \max |v|=1, \quad 0 \leq \theta<2 \pi, \quad \max |\omega|=1.6 .
$$

The corresponding upper bound of the stepsize is obtained from (116)-(118) giving $H_{r}=0.3080$ and $H_{\theta}=0.3850$ so that $H=0.3080$. The initial conditions were chosen as

$$
\mathbf{r}(0)=\left[\begin{array}{ll}
0.8 & 1
\end{array}\right]^{\top}, \quad \boldsymbol{\theta}(0)=\left[\begin{array}{ll}
0 & 1
\end{array}\right]^{\top} .
$$

Figures 3 and 4 show the discrete trajectories for $\theta$ and $r$ respectively for a stepsize $h=0.3$, a value just less than the upper bound. The existence of the pseudo-sliding mode is verified, although the chattering is quite marked. Figures 5 and 6 (see page 16) give the results for the smaller value $h=0.02$. Again the existence of the pseudo-sliding mode is verified, but now with much less chattering. 


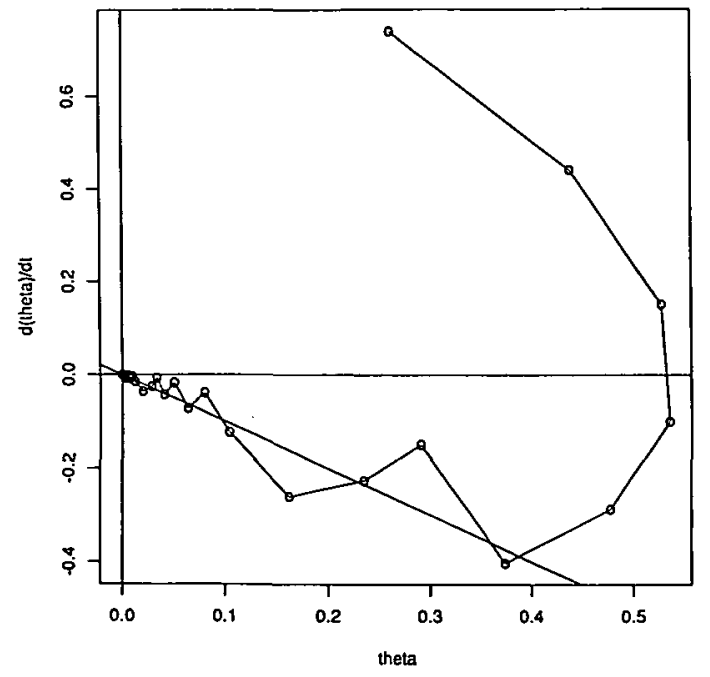

FIGURE 3. Discrete trajectory for the cylindrical robot. The trajectory is for the angle variable with $h=0.3$, just less than the upper bound $H=0.3080$.

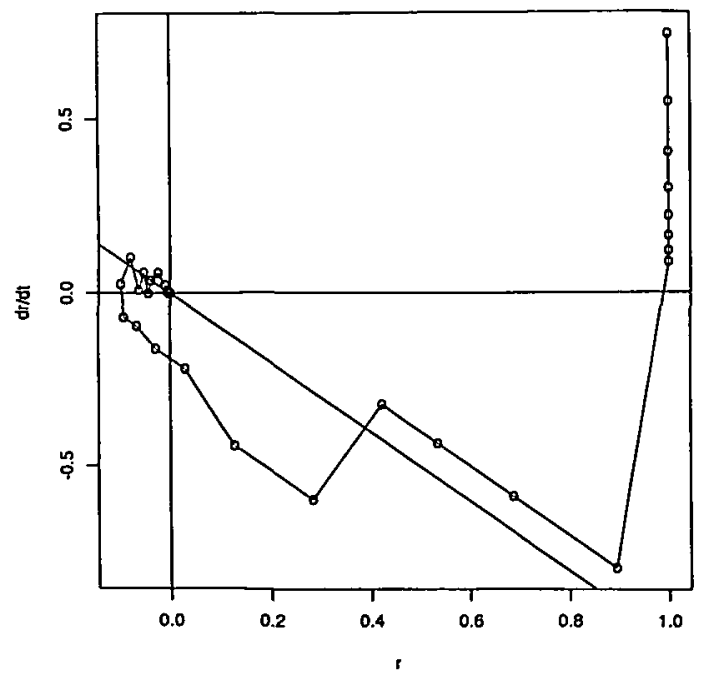

Figure 4. Same as Figure 3 but for the radial variable. 


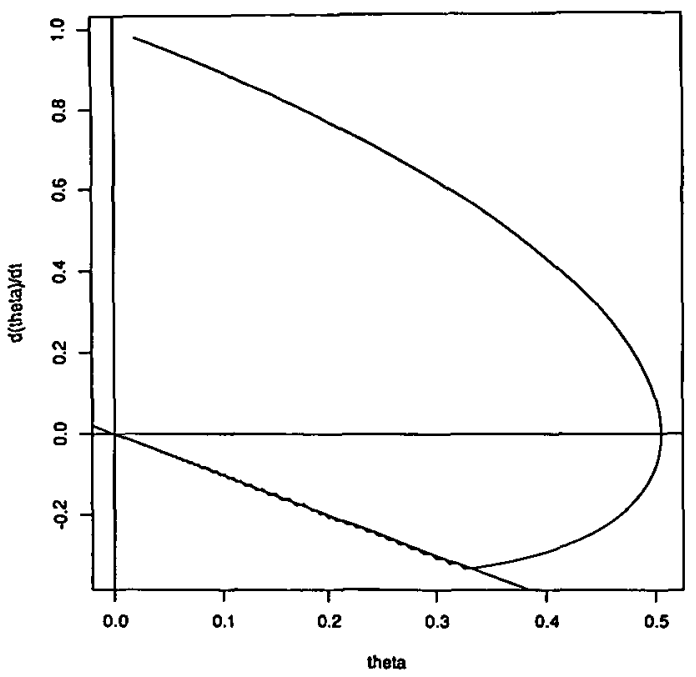

FIGURE 5. Same as Figure 3 but with $h=0.02$.

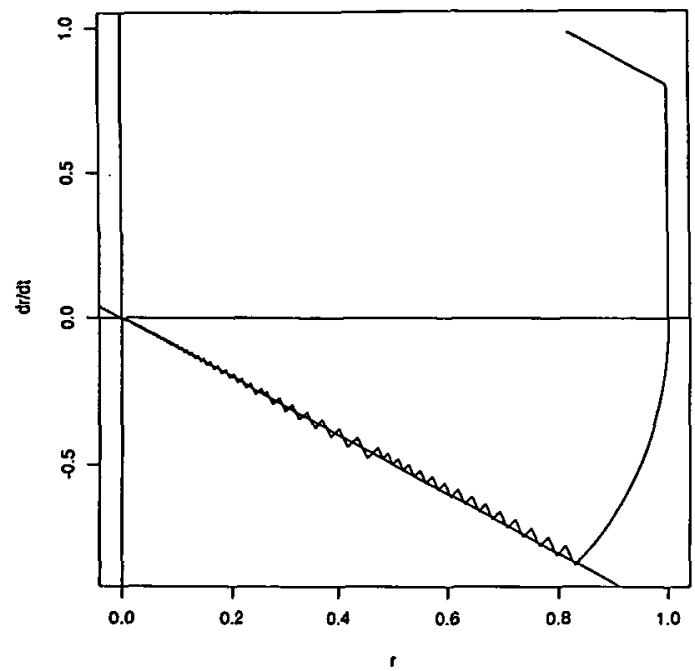

FIGURE 6. Same as Figure 4 but with $h=0.02$. 


\section{Discussion}

The computer control of variable-structure systems has been analysed using zero-order-hold periodic sampling. For a simple two-dimensional system, the resulting discrete system has been solved without approximation, and necessary and sufficient conditions for the existence of a pseudo-sliding mode derived. The analysis has been illustrated with numerical simulations. The method has then been applied to the Neuman-Tourassis discrete model of a cylindrical robot. This model has the advantage of automatically satisfying energy and angular momentum conservation and has been successfully used in robot trajectory and path planning problems [7]. By using hierarchical control of the angle and radius variable in sequence, a sufficient condition limiting the magnitude of the sampling interval has been derived. The analysis has again been illustrated by numerical simulations.

The discrete model of the cylindrical robot is of course an approximation to a continuous model which is itself an approximation to a real world robot. Although the robustness of the control scheme proposed here has been verified by some simulation work, it is now planned to apply the method to a laboratory robot.

\section{Acknowledgement}

The authors are indebted to the Australian Research Council for an award.

\section{References}

[1] K. J. Aström and B. Wittenmark, Computer controlled systems: theory and design (Prentice-Hall, Inglewood Cliffs, 1984).

[2] E. A. Barbashin, Introduction to the theory of stability (Wolters-Noordhoof, Groningen, 1970).

[3] T. A. Bezdovinskaya and E. F. Sabaev, "Study of state space features in variable structure control systems", Autom. Remote Control 7 (1973) 1105-1108.

[4] C. P. Neuman and V. D. Tourassis, "Discrete dynamic robot models", IEEE Trans. Syst. Man Cybern. SMC-15 (1985) 193-204.

[5] J. M. Ortega and W. C. Rheinboldt, Iterative solution of nonlinear equations in several variables (Academic Press, New York, 1970).

[6] R. B. Potts and X. Yu, "Discrete variable structure system with pseudo-sliding mode", J Austral. Math. Soc. Ser. B 32 (1991) 365-376.

[7] H. H. Tan and R. B. Potts, "A discrete path/trajectory planner for robotic arms", $J$. Austral. Math. Soc. Ser. B 31 (1989) 1-28.

[8] V. D. Tourassis and C. P. Neuman, "Inverse dynamics applications of discrete robot models", IEEE Trans. Syst. Man Cybern. SMC-15 (1985) 798-803.

[9] K-K. D. Young, "Controller design for a manipulator using theory of variable structure systems", IEEE Trans. Syst. Man Cybern. SMC-8 (1978) 101-109. 